\title{
Influence of random magnetic field on solar global oscillations: The incompressible $f$-mode
}

\author{
R. Erdélyi, A. Kerekes, and N. Mole \\ Solar Physics \& upper-Atmosphere Research Group, Department of Applied Mathematics, University of Sheffield, \\ The Hicks Building, Hounsfield Road, Sheffield S3 7RH, UK \\ e-mail: [robertus;a.kerekes;n.mole]@sheffield.ac.uk \\ Received 11 June 2004 / Accepted 23 October 2004

\begin{abstract}
The discrepancies between theoretically predicted and observed frequencies of solar global oscillations (e.g. $p$ and $f$-modes) have attracted major attention in the past decades. The $f$-mode is essentially a surface wave hence the mode frequencies are less likely to be influenced by the solar stratification. Most probably then the discrepancies are the result of some near surface mechanism(s) such as interactions with surface or sub-surface magnetic fields and flows. In the following we explore whether the small-scale photospheric magnetic fields, sometimes also called the magnetic carpet, may be part of the explanation for the apparent frequency paradox.
\end{abstract}

Key words. magnetohydrodynamics (MHD) - Sun: helioseismology - Sun: oscillations - Sun: atmosphere Sun: magnetic fields

\section{Introduction}

The solar $f$-mode is usually referred to as an incompressible surface gravity wave with the simple dispersion relation (when the solar surface is considered as a simple density discontinuity) $\omega^{2}=g k_{\mathrm{h}}$, where $g$ is the surface gravitational acceleration and $k_{\mathrm{h}}$ is the horizontal wavenumber. Observations have revealed a significant departure from this parabolic dispersion relation; for spherical low-degree modes (i.e. $l \sim O(100)$ ) the measured frequencies are slightly higher, for high-degree modes several percent lower (Antia 1998; Libbrecht et al. 1990) than the theoretical values. Observations also show a solar-cycle dependence both for $f$ - (Antia et al. 2001) and $p$-modes (Jain et al. 2001). Dziembowski et al. (2001) gave a possible interpretation of part of these measured frequency changes related to variations in the solar radius during the magnetic solar cycle.

The $f$-mode, being a surface wave, is expected to be most sensitive to near-surface effects, so atmospheric magnetism and photospheric and sub-surface flows could well be the cause of the observed discrepancies.

The effect of the large-scale chromospheric magnetic field on global oscillations has already been studied by Campbell \& Roberts (1989) and Evans \& Roberts (1990), who found that the presence of the so-called magnetic canopy increases the $f$-mode frequencies for all wavenumbers. On the other hand, Murawski \& Roberts (1993) showed that small-scale photospheric flows (representing granular motions) can appreciably reduce the $f$-mode frequencies. For a recent review of these atmospheric effects see e.g. Erdélyi (2002). Dziembowski \& Goode (2004) investigated the effects of both large- and smallscale sub-surface dynamics (flows and magnetic fields) while Thompson \& Gough (1990) have carried out detailed calculations for large scale buried magnetic fields.

Similarly to granulation, the photospheric and atmospheric magnetic field is also very dynamic. High resolution magnetograms reveal that outside active regions the solar surface is covered with a mixed polarity network, which is termed magnetic carpet (Title \& Schrijver 1998). The structure of this small-scale field changes rapidly on very short spatial and time scales and flux continuously emerges and disappears nearly homogeneously over the surface. Interestingly, the smallest magnetic structures show no correlation with the solar cycle (Hagenaar et al. 2003), therefore they are believed to originate in a separate, small-scale dynamo process.

Our aim is to investigate the influence of this disorganised, small-scale atmospheric field on the $f$-mode frequencies. In a first approximation the magnetic carpet is modelled as a timeindependent, stochastic field. Since, depending on their spherical degree, some $f$-modes may have a life time comparable to the characteristic replacement time (of the order of tens of hours) of the magnetic carpet, this limits the validity of our study. The magnetic field is taken to be independent of time because first of all we would like to assess the effect of random magnetic field alone and in the case of a time-dependent field one would have to deal with generated flows in the initial state. 


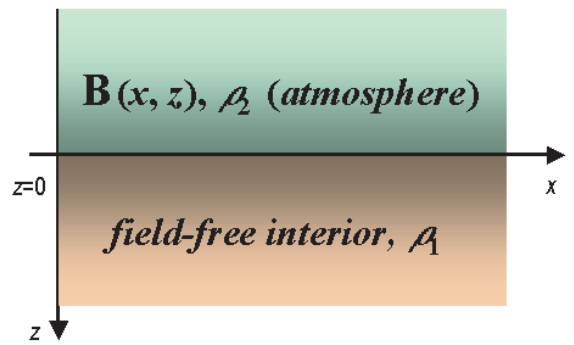

Fig. 1. Model.

\section{The model}

The solar surface region is modelled by a tangential discontinuity. We consider a simple 2-dimensional Cartesian model where the $z=0$ surface corresponds to the solar surface (i.e. the bottom of the photosphere) with the $z$-axis directed downwards so that the gravitational acceleration is in the positive $z$-direction. The magnetic carpet is approximated as a random magnetic field overlaying a field-free interior.

In the initial state the background magnetic field is random and time-independent. This is a good approximation as long as the characteristic time scales of the $f$-modes are much smaller than the replacement time for the magnetic carpet (as discussed above). Both the interior and the atmosphere are taken to be incompressible. No equilibrium flows are present in the system. The latter assumption needs to be handled very carefully because random granular motions are ubiquitous and also influence the global oscillations (Murawski \& Roberts 1993). However, in this study we would like to address the question of whether the magnetic carpet causes relevant and measurable changes to the surface mode frequencies. In reality, of course, both effects, and most probably others as well, are present and should be considered. An initial investigation of coherent magnetic fields and flows was carried out by Erdélyi \& Taroyan (2001). Combined random flows and magnetic fields will be considered in a follow up paper.

The governing equations describing the undisturbed state are

$0=-\nabla p_{0,1}+\rho_{1} g \hat{z}$

in $z>0$, and

$0=-\nabla p_{0,2}+\frac{1}{\mu}\left(\nabla \times \boldsymbol{B}_{0}\right) \times \boldsymbol{B}_{0}+\rho_{2} g \hat{z}$

in $z<0$, where $p_{0,1}$ and $p_{0,2}$ are the fluid pressures in $z>0$ and $z<0$, respectively, $\rho_{1}, \rho_{2}$ are the constant densities and $g$ is the local gravitational acceleration. The boundary condition at $z=0$ is the continuity of total pressure

$p_{0,1}=p_{0,2}+\frac{B_{0}^{2}}{2 \mu}$.

Because of the $\nabla \cdot \boldsymbol{B}=0$ (solenoidal) condition the magnetic field $\boldsymbol{B}$ can always be represented by a vector potential $\boldsymbol{A}$, so that $\boldsymbol{B}=\nabla \times \boldsymbol{A}$. In the current two-dimensional case the vector potential can be chosen, without loss of generality, to have only one component

$\boldsymbol{B}_{0}(x, z)=\nabla \times \boldsymbol{A}_{0}(x, z), \quad \boldsymbol{A}_{0}=\left(0, A_{0}, 0\right)$.
The profile of the initial magnetic field is taken to be

$A_{0}=-z \exp \left(\alpha^{2} z\right) b(x)$.

Thus the field is confined to a layer whose thickness is determined by the $\alpha^{2}$ decay factor. This is a reasonable assumption because not all of the emerged magnetic field reaches the the higher parts of the solar atmosphere. In the case of the solar atmosphere it is plausible to assume that the thickness of such a layer is about a few $100 \mathrm{~km}$ which corresponds to $\alpha^{2}=O\left(10^{-2}\right)\left[\mathrm{km}^{-1}\right]$. The function $b(x)$ is taken to be homogeneous and random with zero mean, so

$\langle b(x)\rangle=0$,

where \langle\rangle denotes the ensemble mean. The magnetic field is then a random field with zero mean which is homogeneous in $x$. The observed global oscillations have extremely small amplitudes compared with the background so they can be described as linear perturbations to the equilibrium state.

\section{Wave perturbation}

Let us now study perturbations to the equilibrium state described in Sect. 2. The governing equations for the dynamical behavior of the perturbations are

$\nabla \cdot v_{1}=0$

$\rho_{1} \frac{\mathrm{d} v_{1}}{\mathrm{~d} t}=-\nabla p_{1}+\rho_{1} g \hat{z}$

in the solar interior region $z>0$, and

$\nabla \cdot \boldsymbol{v}_{2}=0$

$\rho_{2} \frac{\mathrm{d} v_{2}}{\mathrm{~d} t}=-\nabla p_{2}+\frac{1}{\mu}(\nabla \times \boldsymbol{B}) \times \boldsymbol{B}+\rho_{2} g \hat{z}$,

$\boldsymbol{B}_{t}=\nabla \times\left(\boldsymbol{v}_{2} \times \boldsymbol{B}\right)$

in the solar atmospheric region $z<0$. Here the subscript $t$ denotes the partial derivative with respect to $t$. In the presence of a wave motion the generated velocity fields have to satisfy the incompressible condition $\left(\nabla \cdot \boldsymbol{v}_{i}=0, i=1,2\right)$ so they can be represented by the following vector potentials

$$
\begin{aligned}
\boldsymbol{v}_{1}(x, z, t) & =\nabla \times \boldsymbol{\Psi}_{1}(x, z, t), \\
\boldsymbol{\Psi}_{1} & =\left(0, \Psi_{1}, 0\right), \\
\boldsymbol{v}_{2}(x, z, t) & =\nabla \times \Psi_{2}(x, z, t), \\
\boldsymbol{\Psi}_{2} & =\left(0, \Psi_{2}, 0\right) .
\end{aligned}
$$

The boundary conditions at the perturbed surface, $z=\eta(x, t)$, are the kinematic boundary condition

$\eta_{t}=\left(\boldsymbol{v}_{i} \cdot \nabla\right)(z-\eta)$,

and the continuity of total pressure

$p_{1}=p_{2}+\frac{B^{2}}{2 \mu}$.

Since we are interested in surface waves only, where the energy of waves is localized to the surface discontinuity, far away from the interface we require that

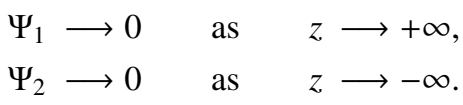




\section{Dispersion relation}

In order to obtain the dispersion relation we integrate the $z$ components of the equations of motion and substitute $z=\eta$. Using the continuity of pressure we can eliminate the pressure terms and the resulting equation connects the scalar functions $\Psi_{1}, \Psi_{2}$ and $A$ (the $y$-components of the corresponding vector potentials) on the perturbed surface $\eta(x, t)$.

It is straightforward to decompose the magnetic and velocity fields into equilibrium and perturbation values, and linearize around the background state. No flow is present in the equilibrium state so $\Psi_{1}=\delta \Psi_{1}, \Psi_{2}=\delta \Psi_{2}, \eta=\delta \eta$, and for the magnetic field $A=A_{0}+\delta A$. Discarding the $\delta$-notations, at $z=0$ we obtain the kinematic boundary conditions

$\eta_{t}=\Psi_{1 x}=\Psi_{2 x}$,

the induction equation

$A_{t}=\Psi_{2 x} b$,

and from the equation of motion

$$
\begin{aligned}
& \kappa \int_{-\infty}^{0} \Psi_{2 x t} \mathrm{~d} z-\int_{\infty}^{0} \Psi_{1 x t} \mathrm{~d} z+a \eta= \\
& \frac{1}{\mu \rho_{1}} \int_{-\infty}^{0} \mathrm{e}^{\alpha^{2} z}\left(1+\alpha^{2} z\right)\left(b A_{x x}-b_{x} A_{x}\right) \mathrm{d} z \\
& \quad+\frac{1}{\mu \rho_{1}} \int_{-\infty}^{0} \mathrm{e}^{\alpha^{2} z} z\left(b_{x x} A_{z}-b_{x} A_{x z}\right) \mathrm{d} z .
\end{aligned}
$$

Because of the presence of the random fields we divide the wave fields into random and coherent components

$$
\begin{array}{lc}
A=\langle A\rangle+A^{\prime} & \left\langle A^{\prime}\right\rangle=0, \\
\Psi_{1}=\left\langle\Psi_{1}\right\rangle+\Psi_{1}^{\prime} & \left\langle\Psi_{1}^{\prime}\right\rangle=0, \\
\Psi_{2}=\left\langle\Psi_{2}\right\rangle+\Psi_{2}^{\prime} & \left\langle\Psi_{2}^{\prime}\right\rangle=0, \\
\eta=\langle\eta\rangle+\eta^{\prime} & \left\langle\eta^{\prime}\right\rangle=0 .
\end{array}
$$

Substituting Eqs. (23)-(26) into Eqs. (20)-(22) and taking ensemble averages we obtain the equations for the coherent field at $z=0$. Subtracting these equations from Eqs. (20)-(22) and using the the binary collision approximation (Howe 1971) we arrive at the equations governing the evolution of the random field at $z=0$. From the induction equation we obtain

$\langle A\rangle_{t}=\left\langle b \Psi_{2 x}^{\prime}\right\rangle$,

$A_{t}^{\prime}=b\left\langle\Psi_{2 x}\right\rangle$,

from the kinematic boundary condition

$\langle\eta\rangle_{t}=\left\langle\Psi_{1 x}\right\rangle=\left\langle\Psi_{2 x}\right\rangle$,

$\eta_{t}^{\prime}=\Psi_{1 x}^{\prime}=\Psi_{2 x}^{\prime}$,

and from the equation of motion

$$
\begin{gathered}
\kappa \int_{-\infty}^{0}\left\langle\Psi_{2}\right\rangle_{x t} \mathrm{~d} z-\int_{\infty}^{0}\left\langle\Psi_{1}\right\rangle_{x t} \mathrm{~d} z+a\langle\eta\rangle= \\
\frac{1}{\mu \rho_{1}} \int_{-\infty}^{0} \mathrm{e}^{\alpha^{2} z}\left(1+\alpha^{2} z\right)\left(\left\langle b A_{x x}\right\rangle-\left\langle b_{x} A_{x}\right\rangle\right) \mathrm{d} z \\
+\frac{1}{\mu \rho_{1}} \int_{-\infty}^{0} \mathrm{e}^{\alpha^{2} z} z\left(\left\langle b_{x x} A_{z}\right\rangle-\left\langle b_{x} A_{x z}\right\rangle\right) \mathrm{d} z,
\end{gathered}
$$

and

$$
\begin{aligned}
& \kappa \int_{-\infty}^{0} \Psi_{2 x t}^{\prime} \mathrm{d} z-\int_{\infty}^{0} \Psi_{1 x t}^{\prime} \mathrm{d} z+a \eta^{\prime}= \\
& \frac{1}{\mu \rho_{1}} \int_{-\infty}^{0} \mathrm{e}^{\alpha^{2} z}\left(1+\alpha^{2} z\right)\left(b\langle A\rangle_{x x}-b_{x}\langle A\rangle_{x}\right) \mathrm{d} z \\
& +\frac{1}{\mu \rho_{1}} \int_{-\infty}^{0} \mathrm{e}^{\alpha^{2} z} z\left(b_{x x}\langle A\rangle_{z}-b_{x}\langle A\rangle_{x z}\right) \mathrm{d} z
\end{aligned}
$$

It is straightforward to write the perturbation quantities in Fourier form, so that

$$
\begin{aligned}
& A^{\prime}(x, z, t)=F^{-1}\left\{\mathrm{e}^{|k| z-i \omega t} \hat{A}(k)\right\} \\
& =\frac{1}{2 \pi} \int_{-\infty}^{\infty} \hat{A}(k) \mathrm{e}^{|k| z} \mathrm{e}^{i(k x-\omega t)} \mathrm{d} k, \\
& \langle A\rangle(x, z, t)=F^{-1}\left\{\mathrm{e}^{|k| z-i \omega t} \bar{A}(k)\right\} \\
& \Psi_{2}^{\prime}(x, z, t)=F^{-1}\left\{\mathrm{e}^{|k| z-i \omega t} \hat{\Psi}_{2}(k)\right\}, \\
& \left\langle\Psi_{2}\right\rangle(x, z, t)=F^{-1}\left\{\mathrm{e}^{|k| z-i \omega t} \bar{\Psi}_{2}(k)\right\}, \\
& \Psi_{1}^{\prime}(x, z, t)=F^{-1}\left\{\mathrm{e}^{-|k| z-i \omega t} \hat{\Psi}_{1}(k)\right\}, \\
& \left\langle\Psi_{1}\right\rangle(x, z, t)=F^{-1}\left\{\mathrm{e}^{-|k| z-i \omega t} \bar{\Psi}_{1}(k)\right\}, \\
& \eta^{\prime}(x, t)=F^{-1}\left\{\mathrm{e}^{-i \omega t} \hat{\eta}(k)\right\}, \\
& \langle\eta\rangle(x, t)=F^{-1}\left\{\mathrm{e}^{-i \omega t} \bar{\eta}(k)\right\} .
\end{aligned}
$$

We introduce the following new notations

$\kappa=\rho_{2} / \rho_{1} \quad$ density ratio,

$R_{0}\left(x-x_{1}\right)=\left\langle b(x) b\left(x_{1}\right)\right\rangle$ correlation function,

$\hat{R}_{0}\left(k-k_{1}\right)$ Fourier transformed correlation function.

Applying Fourier transforms to Eqs. (27)-(32) leads to the dispersion relation

$$
\begin{aligned}
& (1+\kappa) \omega^{2} \operatorname{sgn}(k)-(1-\kappa) g k= \\
& \quad \frac{k^{2}}{\mu \rho_{1}} \int_{-\infty}^{\infty} \frac{\left|k_{1}\right|\left(2 k_{1}-k\right) \hat{R}_{0}\left(k-k_{1}\right)}{\left(\alpha^{2}+\left|k_{1}\right|\right)^{2}} \mathrm{~d} k_{1} .
\end{aligned}
$$

Let us introduce a Gaussian correlation function and its Fourier transform

$$
\begin{aligned}
& R_{0}(x)=\sigma_{0}^{2} \mathrm{e}^{-x^{2} / 4 l_{c}^{2}}, \\
& \hat{R}_{0}(k)=\int_{-\infty}^{\infty} R_{0}(x) \mathrm{e}^{-i k x} \mathrm{~d} x=\sigma_{0}{ }^{2} \frac{l_{\mathrm{c}}}{\sqrt{\pi}} \mathrm{e}^{-k^{2} l_{\mathrm{c}}^{2}},
\end{aligned}
$$

where $\sigma_{0}$ and $l_{\mathrm{c}}$ are the characteristic strength and length-scale of the magnetic field. Substitution of the correlation function into Eq. (41) yields

$$
\begin{aligned}
& (\kappa+1) \omega^{2} \operatorname{sgn}(k)-(1-\kappa) g k= \\
& \quad \frac{\sigma_{0}^{2} k^{2} l_{\mathrm{c}}}{\mu \rho_{1} \sqrt{\pi}} \int_{-\infty}^{\infty} \frac{\left|k_{1}\right|\left(2 k_{1}-k\right)}{\left(\alpha^{2}+\left|k_{1}\right|\right)^{2}} \mathrm{e}^{-\left(k-k_{1}\right)^{2} l_{\mathrm{c}}^{2}} \mathrm{~d} k_{1} .
\end{aligned}
$$

In the field-free case the right-hand side of the dispersion equation is zero returning the standard dispersion relation for surface waves propagating along the interface between two semi-infinite layers with constant densities. In the absence of an atmospheric magnetic field we obtain

$\omega_{0}^{2}=\frac{1-\kappa}{1+\kappa} g k$. 


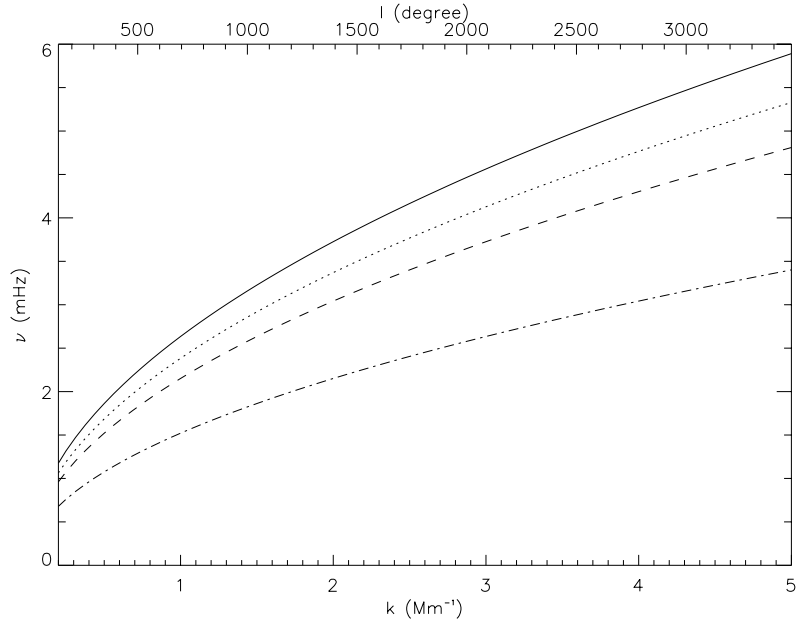

Fig. 2. The $f$-mode frequencies in the absence of magnetic field for different values of the density ratio; $\kappa=0$ corresponds to the wellknown water wave result $\omega^{2}=g k$ (solid), $\kappa=0.1$ (dotted), $\kappa=0.2$ (dashed) and $\kappa=0.5$ (dash-dot).

The random magnetic field results in a correction to this dispersion relation where the correction is given by the RHS of Eq. (44).

The fact that our model has an atmosphere with a small though non-zero density has a considerable effect (at least when comparing it to the corrections due to the magnetic carpet) on the $f$-mode frequencies. Figure 2 shows the obtained frequencies $(v=\omega / 2 \pi)$ of the $f$-mode as the functions of the wavenumber $k$ in the case of a magnetic field-free atmosphere for different values of the density ratio at the tangential discontinuity. The corrections due to the presence of an atmosphere could be of the order of $\mathrm{mHz}$ or even more. This has to be kept in mind when comparing results with observations. In Fig. 2 we also indicate the spherical degree $l$, which is related to $k$ by the expression

$k^{2}=\frac{l(l+1)}{R_{\odot}^{2}}$,

where $R_{\odot}$ denotes the solar radius. Of course, we are aware that modelling the transition from solar interior to the atmosphere with a tangential discontinuity may be far from reality. A continuous (i.e. stratified) atmosphere may be a better approach when comparing results with observations. However, as pointed out before, our aim is to establish the effects of the magnetic carpet only, and introducing a more complex atmosphere would make the analysis much more difficult without clarifying this point.

\section{Numerical results}

In order to analyze the dispersion relation (44) and to obtain the frequency shifts caused by the atmospheric magnetic carpet we introduce the following non-dimensional variables:

$K=k l_{\mathrm{c}}, \quad K_{1}=k_{1} l_{\mathrm{c}}$

denote the dimensionless wavenumbers,

$p=\alpha^{2} l_{\mathrm{c}}$ is the dimensionless decay factor,

$\Omega^{2}=\left(\frac{1+\kappa}{1-\kappa}\right) \frac{l_{\mathrm{c}}}{g} \omega^{2}$

is the dimensionless frequency, and finally

$\frac{\sigma_{0}^{2}}{\mu \rho_{1}}=\kappa \frac{\sigma_{0}^{2}}{\mu \rho_{2}}=\kappa v_{\mathrm{Ar}}^{2}$,

where $v_{\text {Ar }}$ denotes the characteristic Alfvén speed of the random field. Equation (44) can be written in its dimensionless form

$\Omega^{2} \operatorname{sgn}(K)-K=$

$\frac{v_{\mathrm{Ar}}^{2}}{g l_{\mathrm{c}} \sqrt{\pi}}\left(\frac{\kappa}{1-\kappa}\right) K^{2} \int_{-\infty}^{\infty} \frac{\left|K_{1}\right|\left(2 K_{1}-K\right)}{\left(p+\left|K_{1}\right|\right)^{2}} \mathrm{e}^{-\left(K-K_{1}\right)^{2}} \mathrm{~d} K_{1}$.

The dispersion relation is solved numerically in the form

$\Omega^{2} \operatorname{sgn}(K)-K=2 \frac{v_{\mathrm{Ar}}^{2}}{g l_{\mathrm{c}} \sqrt{\pi}}\left(\frac{\kappa}{1-\kappa}\right) K^{2} \mathrm{e}^{-K^{2}}\left(2 I_{1}-K I_{2}\right)$,

where

$$
\begin{aligned}
I_{1}= & \frac{\sqrt{\pi}}{2} \mathrm{e}^{K^{2}} \operatorname{erf}(K)-2 p \int_{0}^{\infty} \frac{\mathrm{e}^{-s^{2}}}{s+p} \sinh (2 K s) \mathrm{d} s \\
& +p^{2} \int_{0}^{\infty} \frac{\mathrm{e}^{-s^{2}}}{(s+p)^{2}} \sinh (2 K s) \mathrm{d} s,
\end{aligned}
$$

and

$$
\begin{aligned}
I_{2}= & \int_{0}^{\infty} \frac{\mathrm{e}^{-s^{2}}}{s+p} \cosh (2 K s) \mathrm{d} s \\
& -p \int_{0}^{\infty} \frac{\mathrm{e}^{-s^{2}}}{(s+p)^{2}} \cosh (2 K s) \mathrm{d} s .
\end{aligned}
$$

Numerical results of the corrections to the dispersion relation are displayed in Figs. 3-5. On these figures we plot the frequency correction $\Delta v=v-v_{0}$ as a function of the dimensional wave number for various key parameters determining the equilibrium (e.g. random Alfvén speed, $v_{\mathrm{Ar}}$; density ratio $\kappa$; dimensionless decay factor $p$, etc.).

It is apparent from these figures that the presence of the random magnetic field results in a frequency increase for all wavenumbers. Similar results were obtained by Campbell \& Roberts (1989) and Evans \& Roberts (1990) who investigated the effects of the magnetic canopy (modeled as uniform or exponentially decaying magnetic field) on global oscillations. The $f$-mode can only be identified securely in the range $100<$ $l<3000$. For very small values of $l$ the frequencies cannot be measured reliably because the modes do not have sufficient power. For very large values of $l$ the damping time of the mode becomes comparable to the period of the oscillation, therefore the mode cannot be considered to exist as a normal standing wave (Antia \& Basu 1999). Positive frequency shifts were only measured for the lowest observable degrees; the dominant effect is that the $f$-mode frequencies are appreciably reduced for intermediate and higher degrees (Antia 1998). Apparently, magnetic fields alone cannot be responsible for the observed discrepancies. The complex behavior of the frequency shifts is 


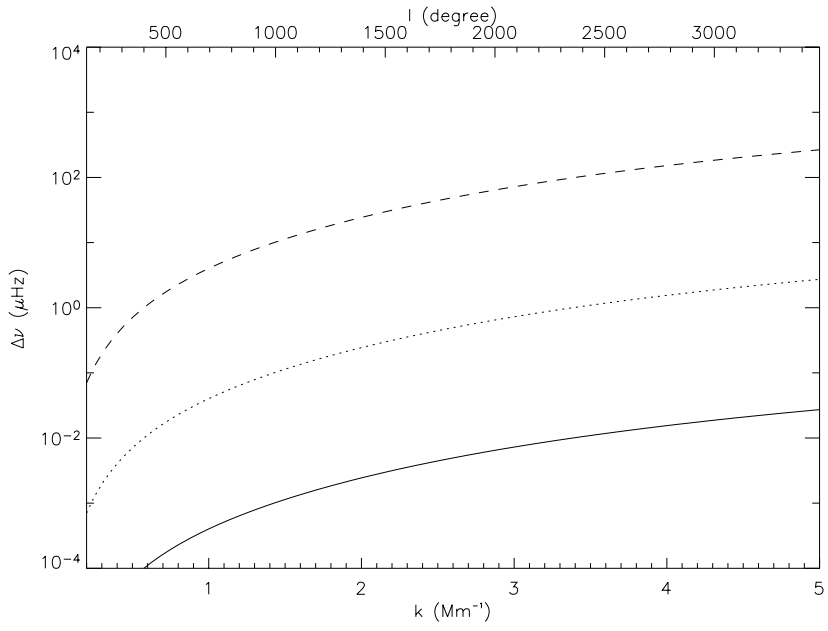

Fig. 3. Frequency correction $\Delta v=v-v_{0}$ as function of the wavenumber for $v_{\mathrm{Ar}}=0.1 \mathrm{~km} \mathrm{~s}^{-1}$ (solid), $v_{\mathrm{Ar}}=1 \mathrm{~km} \mathrm{~s}^{-1}$ (dotted) and $v_{\mathrm{Ar}}=$ $10 \mathrm{~km} \mathrm{~s}^{-1}$ (dashed) with $l_{\mathrm{c}}=10^{3} \mathrm{~km}, \kappa=0.2, p=5$.

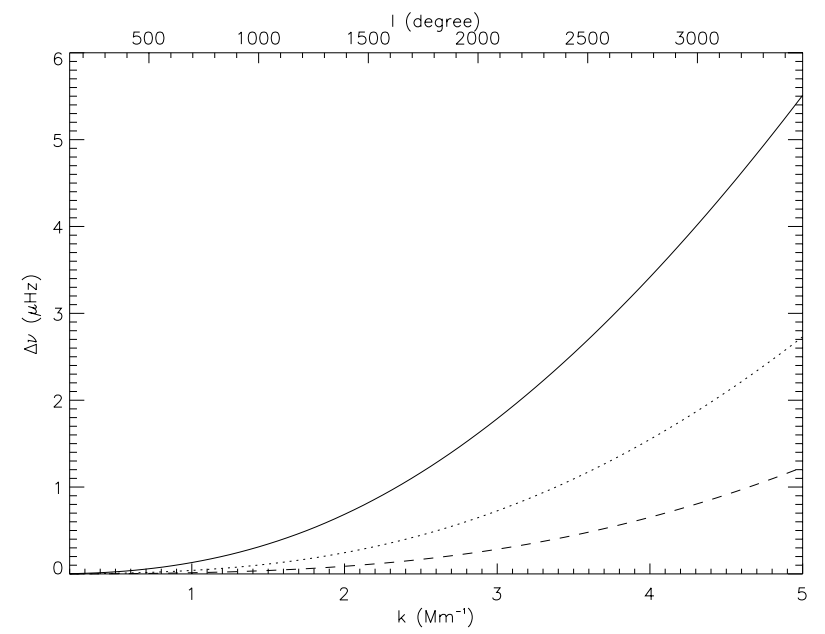

Fig. 4. Frequency correction $\Delta v=v-v_{0}$ as function of the wavenumber for $p=2$ (solid), $p=5$ (dotted) and $p=10$ (dashed), with $l_{\mathrm{c}}=10^{3} \mathrm{~km}, v_{\mathrm{Ar}}=1 \mathrm{~km} \mathrm{~s}^{-1}, \kappa=0.2$.

probably the result of several competing processes that dominate in different frequency ranges.

First let us investigate how the frequency shifts are influenced by the random Alfvén speed $\left(v_{\mathrm{Ar}}\right)$ that could be a proxy measure to establish the influence of the intensity of the magnetic carpet (see Fig. 3). Due to observational difficulties the small-scale field cannot be measured accurately because the diameter of the individual flux tubes is probably much smaller than the resolution of MDI (on-board SOHO) or ground-based (e.g. La Palma) data. The MDI instrument produces magnetograms in two modes: full-disc magnetograms with a pixel size of $2^{\prime \prime}$ at disc center and high-resolution magnetograms with a pixel size of $0.61^{\prime \prime}$ (Hagenaar 2001). The average flux density, which is the measured flux divided by the corresponding area, can only give a lower limit for the value of the magnetic field concentrated in such thin flux tubes (i.e. this is the so-called filling factor problem). On the other hand, it has been established that the sound speed and the Alfvén

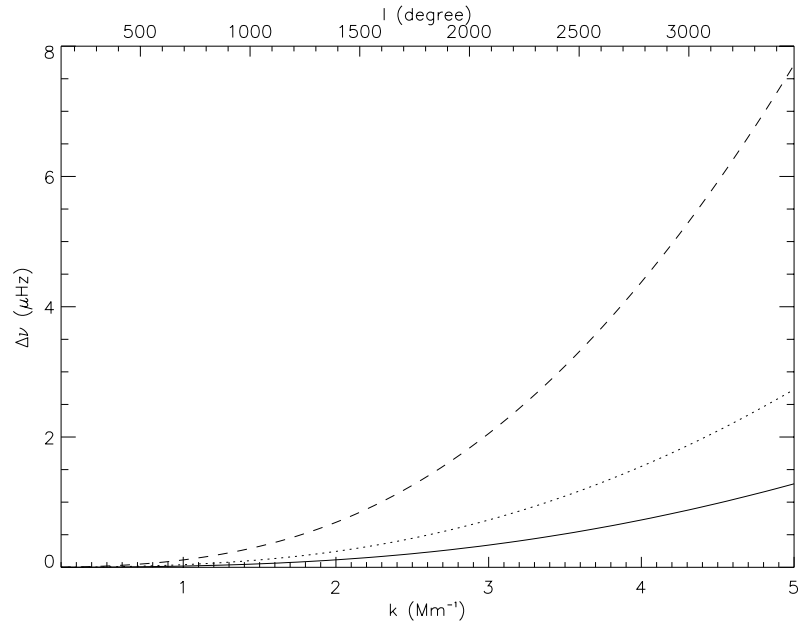

Fig. 5. Frequency correction $\Delta v=v-v_{0}$ as function of the wavenumber for $\kappa=0.1$ (solid), $\kappa=0.2$ (dotted) and $\kappa=0.5$ (dashed), with $l_{\mathrm{c}}=10^{3} \mathrm{~km}, v_{\mathrm{Ar}}=1 \mathrm{~km} \mathrm{~s}^{-1}, p=5$.

speed are of the same order in the lower solar atmosphere, so we considered $v_{\mathrm{Ar}}$ values between 0.1 and $10 \mathrm{~km} \mathrm{~s}^{-1}$. It is clear from the plots that a higher random Alfvén speed (say above $1 \mathrm{~km} \mathrm{~s}^{-1}$ ) would result in unrealistically strong frequency shifts.

Next let us investigate how the frequency shifts are influenced by the thickness of the magnetic carpet. In the current working model the random magnetic field is confined to a layer whose thickness is prescribed by the dimensionless decay parameter $p$. The small-scale field is expected to dominate in a layer a few $100 \mathrm{~km}$ thick in the photosphere, which places $p$ between 2 and 10. Figure 4 shows the variations of the correction term within this range. We find the stronger the decay (i.e. the thinner the layer), the less the surface wave frequency is shifted. To fine tune this parameter, $p$, we need observations that would measure or give indications about the characteristic height of the magnetic carpet. We are convinced that such observations will be the centre of interest in future solar surface studies.

Finally, we also find that the frequency correction largely depends on the ratio of the density of the atmosphere and the density of the interior, apparently, for high contrast the correction is decreased (see Fig. 5). Of course we are aware that a tangential discontinuity is a fairly simple representation of the density changes between the solar interior and the solar atmosphere. We shall remove this working constraint by introducing a continuous boundary layer in a future study.

\section{Conclusions}

Solar global oscillations turned out to be of fundamental importance when one wishes to gain information about the interior part of the Sun. Depending on the spherical degree of such modes the various modes penetrate to various depths under the sub-surface photospheric layer, sampling information about the physical conditions along their ray paths. The solar global $f$ - or 
fundamental mode is an incompressible surface wave which is mainly sensitive to the changes in the physical parameters close to the top of the solar surface.

The solar surface is a very dynamic, rapidly changing layer where magnetic flux is emerging and disappearing. This continuously varying magnetic field, called the magnetic carpet, has a key role in the dynamics of the lower solar atmosphere. In this paper we investigated the effect of the magnetic carpet, present at the lower boundary of the solar atmosphere, on solar global oscillations. In particular, to demonstrate the effect is there and should be investigated in detail we illustrated in a simple working model how the magnetic carpet can cause measurable changes in the frequencies of the global $f$-modes.

We found that a time-independent random magnetic field can significantly increase the $f$-mode frequencies in contrast with random steady flows which tend to have an opposite effect (Murawski \& Roberts 1993). Observations of $p$-modes show a more complex picture, and it is found that for some spherical degrees there is actually a frequency decrease as a function of the sunspot number - a good proxy for magnetic flux. In reality both magnetic and velocity fields are very dynamic at the solar surface, therefore studying their interaction could be of crucial importance in interpreting the observed frequency shifts.

Our knowledge of the small-scale solar magnetic field is rather limited and so far there is no information available about any possible connection between the solar cycle and the evolution of the magnetic carpet. If such a correlation would be found observationally the correlation could be simply tested against frequency shifts of solar global oscillations. On the other hand, observations (Antia et al. 2001; Jain et al. 2001) suggest that the $f$-mode may show some correlations with the solar activity index, though it is more significant in the case of the $p$-modes. One way to assess the observed changes with the solar cycle could be to include a coherent solar cycle dependent background field in the model. Also, in order to estimate the frequency changes of $p$-modes the current working model has to be improved by allowing e.g. temperature dependence in the solar interior part $(z>0)$.
We are also aware that in order to be able to use an analytical approach we tried to keep our mathematical model as simple as possible. However, the assumptions may have removed some of the substantial physical effects (e.g. Alfvén or magnetoacoustic waves present in the system instead of the pure acoustic oscillations) which may be corrected in future studies.

Acknowledgements. The authors acknowledge the financial support by PPARC (UK), the Royal Society and the NFS Hungary (OTKA, TO43741). R.E. acknowledges M. Kéray for patient encouragement.

\section{References}

Antia, H. M. 1998, A\&A, 330, 336

Antia, H. M., \& Basu, S. 1999, ApJ 519, 400

Antia, H. M., Basu, S., Pintar, J., \& Schou, J. 2001, in Helio- and Asteroseismology at the Dawn of the Millennium, ed. A. Wilson ESA SP-464, 27

Campbell, W. R., \& Roberts, B. 1989, ApJ, 338, 538

Dziembowski, W. J., \& Goode, P. R. 2004, ApJ, 600, 464

Dziembowski, W. J., Goode, P. R., \& Schou, J. 2001, ApJ, 553, 897

Erdélyi, R. 2002, in Solar Variability: From Core to Outer Frontiers, ed. A. Wilson, ESA SP-506, 869

Erdélyi, R., \& Taroyan, Y. 2001, in Recent Insights into the Physics of the Sun and Heliosphere: Highlights from SOHO and other Space Missions, ed. P. Brekke, et al., IAU Symp., 203, 208

Evans, D., \& Roberts, B. 1990, ApJ, 356, 704

Hagenaar, H. J. 2001, ApJ, 555, 448

Hagenaar, H. J., Schrijver, C. J., \& Title, A. M. 2003, ApJ, 584, 1107

Howe, M. S. 1971, J. Fluid Mech., 45, 769

Jain, K., Tripathy, S. C., \& Bhatnagar, A. 2001, in Helio- and Asteroseismology at the Dawn of the Millennium, ed. A. Wilson, ESA SP-464, 95

Libbrecht, K. G., Woodard, M. F., \& Kaufman, J. M. 1990, ApJS, 74, 1129

Murawski, K., \& Roberts, B. 1993, A\&A, 272, 60

Title, A. M., \& Schrijver, C. J. 1998, The Tenth Cambridge Workshop on Cool Stars, Stellar Systems and the Sun, ed. R. A. Donahue, \& J. A. Bookbinder, ASP Conf. Ser., 154, 345

Thomspon, M. J., \& Gough, D. O. 1990, MNRAS, 242, 25 